\title{
Rectovaginal Fistula of Different Etiologies: Clinical Case Reports
}

\author{
Mitra P, Mukherjee S, Hassan H, Soreng PS, Adhikari S, Roy TK \\ Department of Obstetrics and Gynecology, Medical College Hospital, Kolkata, India
}

\begin{abstract}
We report three cases of recto vaginal fistula (RVF) due to different etiologies. First case was due to sexual trauma (vaginal intercourse) which is not very common. The second case was due to obstetric trauma (the most common etiology of RVF) and the third case was caused by chronic lower genito-urinary infection in a HIV positive lady. We have included the successful surgical procedures of first two cases. But the repairing of the third case was failed hence planned to operate after six months.
\end{abstract}

Keywords: Obstetric trauma, recto vaginal fistula, recto vaginal fistula repair, sexual trauma

\section{INTRODUCTION}

Recto-Vaginal Fistula (RVF) is defined as epithelium-lined tract between the recto sigmoid or anal canal and the vagina or perineum through the recto-vaginal septum, accounts for less than $5 \%$ of all anorectal fistula, usually present as passage of flatus or stool from the vagina, proctitis, fecal incontinence, vaginitis etc. This poses a formidable therapeutic challenge for surgeons.

\section{CASE 1}

A 30 years parous lady presented in the Gyne OPD of Medical College Hospital, Kolkata with complains of continuous passage of flatus and loose stool per vaginum and pain in the lower abdomen for last two weeks. Two and half weeks back she first noticed immediate post coital bleeding on two subsequent occasions which were minimal and subsided spontaneously. After three days, she had the above mentioned symptoms. The vaginal intercourse was performed in dorsal position and not under influence of any drug, narcotic, alcohol or aphrodisiac. She didn't have dyspareunia, abnormal vaginal bleeding or discharge, abnormal rectal bleeding or discharge, constipation and urinary incontinence in the past. She is para $3+0$, all delivered vaginally (only first one with episiotomy). Her last child birth was six months back. There were no histories of prolonged labor, operative or traumatic vaginal delivery. Her past medical and surgical histories were non-significant.

Systemic and per abdomen examination findings were within normal limit. On per speculum examination cervix looked healthy with normal discharge. Per vagina examination revealed a defect sized about $1.5 \times 1.5 \mathrm{~cm}$ in the posterior vaginal wall noted about $2.5 \mathrm{~cm}$ above the introitus, extending up to rectum (Fig 1). Uterus and adnexa were normal. On per rectal examination the above mentioned vaginal findings were corroborated (Fig 2). There were no other anomalies. Hence diagnosis of

\section{CORRESPONDENCE}

Dr. Parthasarathi Mitra

RMO cum clinical tutor

Department of Obstetrics and Gynaecology

Medical College Hospital, Kolkata

Phone: 9432977497

Email : parthasmdoc@rediffmail.com 
low recto-vaginal fistula (traumatic) was made and the fistula was repaired transvaginally in two layers. Rectal wall mucosal defect closed by interrupted stitches and the seromuscular layer by continuous stitches with 3-0 vicryl. Vaginal gap was closed by continuous suture with 3-0 vicryl. Per operatively one unit of whole blood was transfused. The post operative period was uneventful. Patient became continent of flatus and faeces. Her vaginal and rectal examinations were normal in follow-up.

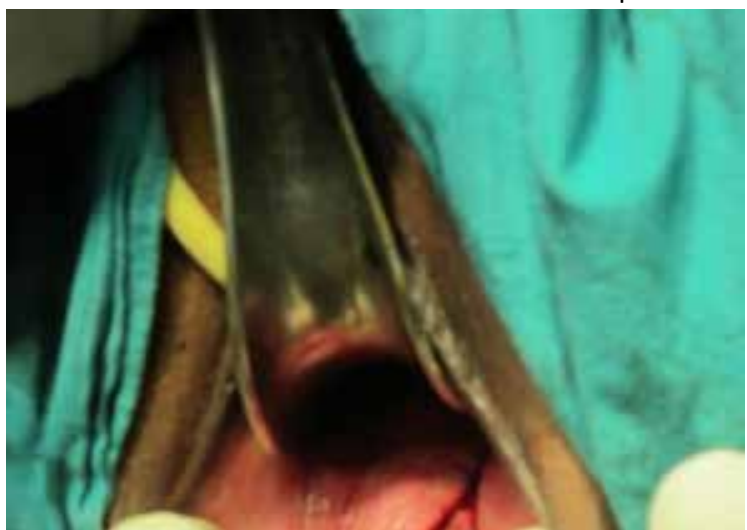

Figure 1. Rent in the posterior vaginal wall due to coital injury

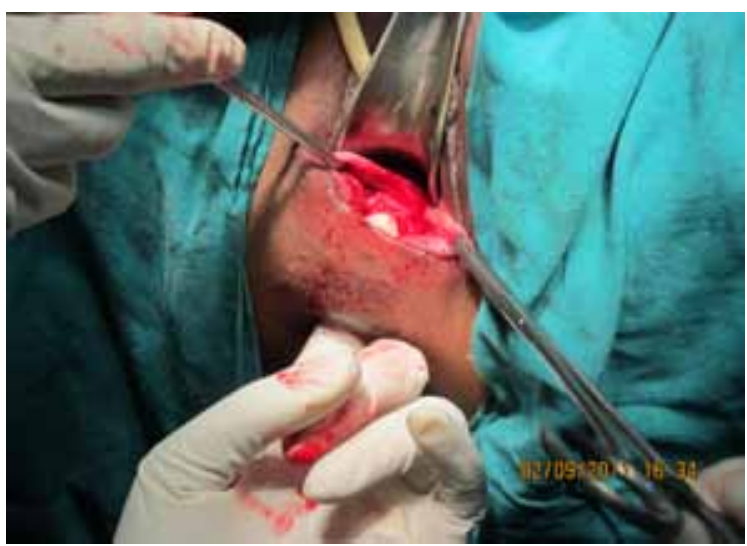

Figure $2.5 \times 1.5 \mathrm{~cm}$ rent extending upto rectum admits tip of the examining finger through rectum

\section{CASE 2}

A patient 32 years, para $2+0$ lady admitted with complaints of intermittent passage of loose stool through the birth canal for last eleven years i.e. immediately after the birth of her last child. Her first child birth was normal vaginal delivery with episiotomy at rural hospital. But she had prolonged labour during second delivery which was conducted at home by local dai. There was extensive vaginal and perineal laceration, hence she was taken to rural hospital and undergone surgical repair. On sixth post partum day she noticed involuntary passage of loose stool per vaginum which was subsided spontaneously. During the last eleven years she was having the above mentioned symptoms intermittently. Her menstrual cycles were normal. She has no significant surgical history except history of appendicectomy seventeen years back. She is hypertensive otherwise she has no other medical illnesses

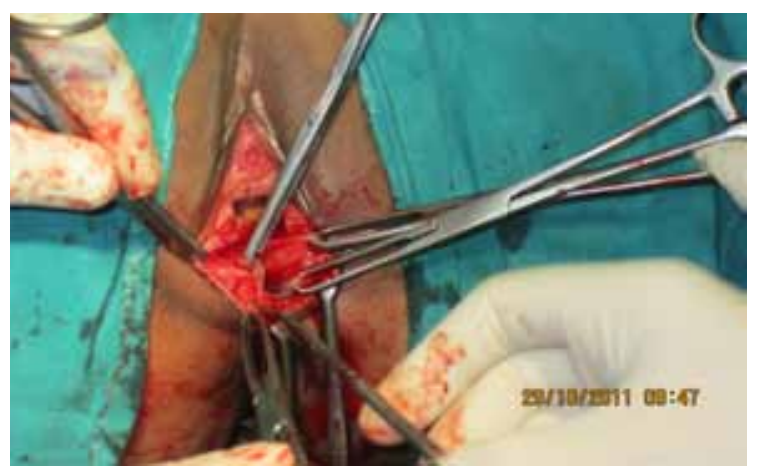

Figure 3. Midline crater in the posterior vaginal wall contiguous with the rectum causing RVF in a case of old child birth injury

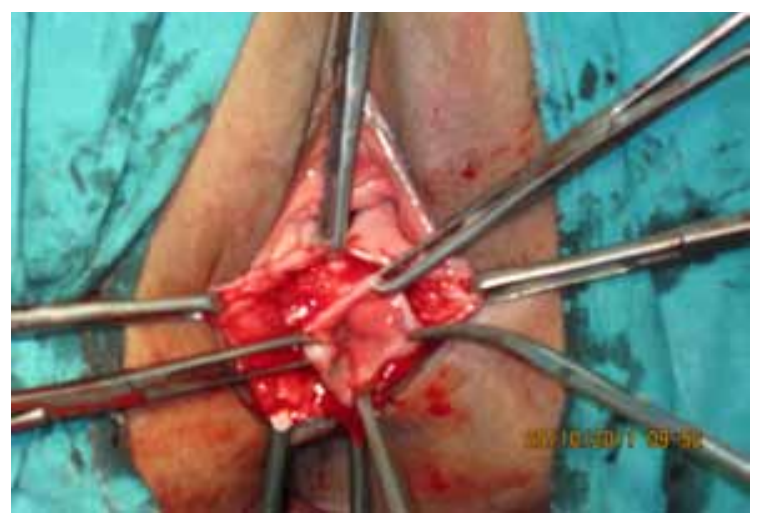

Figure 4. Vagina mobilized from the underlying rectal wall with sharp dissection and excission of entire fistulous tract including a small rim of the rectal mucosa

Her systemic and abdominal examinations were normal except elevated BP. Speculum examination of vagina showed dark red epithelium of fistulous opening in the posterior vaginal wall. Cervix looked healthy with normal discharge. On bimanual examination, two compartments in the vagina with a crater of $2 \times 2 \mathrm{~cm}$ were noted in the midline of posterior vaginal wall near the apex which was continuous with the rectum (Fig 3). Uterus was small, bilateral fornices and POD was clear. Digital rectal exam reveals evidence of fibrosis and induration around fistulous tract without rectal stricture and haematochazia. Her anal sphincters were intact. RVF was repaired transvaginally. A circular incision made around the fistulous opening to separate and widely mobilise the vagina from the underlying rectal wall with sharp dissection (Fig 4). Entire fistulous tract was excised including a small rim of the rectal mucosa. Rectal wall was repaired including the muscularis and submucosa with 3-0 vicryl. A second layer was repaired inverting the initial suture line into the rectum and the vaginal wall with 3-0 vicryl. Patient became continent of flatus and faeces in the post operative period. Follow-up pelvic and rectal examinations were normal. 


\section{CASE 3}

A 37 years parous lady presented with complaints of stool coming from vagina for last one month specially during passage of loose stool. She was para $2+0$, both delivered vaginally with episiotomy at home 20 and 17 years back respectively. There was no history of prolonged labour or operative or traumatic vaginal delivery. The post partum periods on both occasions were uneventful. Her menstrual history was normal. Occasionally she had excessive white vaginal discharge, dysuria and pruritus vulvae. Though she had undergone repeated treatment but never cured permanently. Six months ago she was detected HIV positive and was on anti retroviral therapy (ART). HBV, HCV and VDRL was nonreactive. No other significant medical and surgical history was recorded.

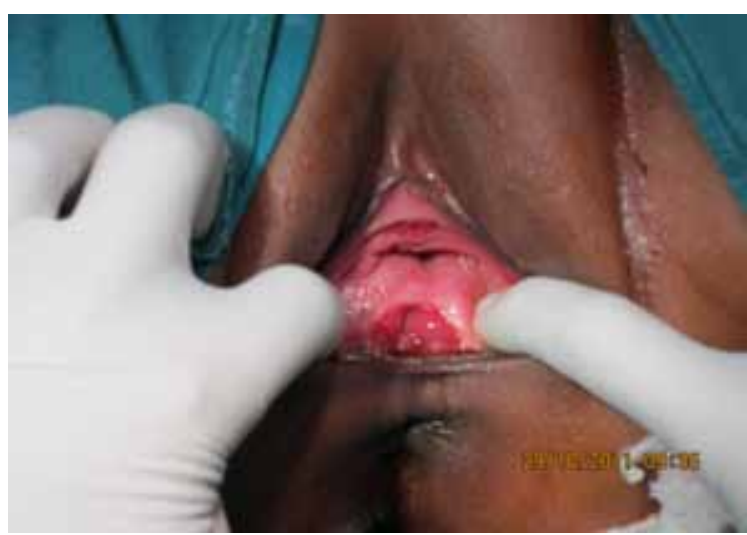

Figure 5. RVF of $2 \times 2 \mathrm{~cm}, 1.5 \mathrm{~cm}$ above the introitus in a case of chronic PID

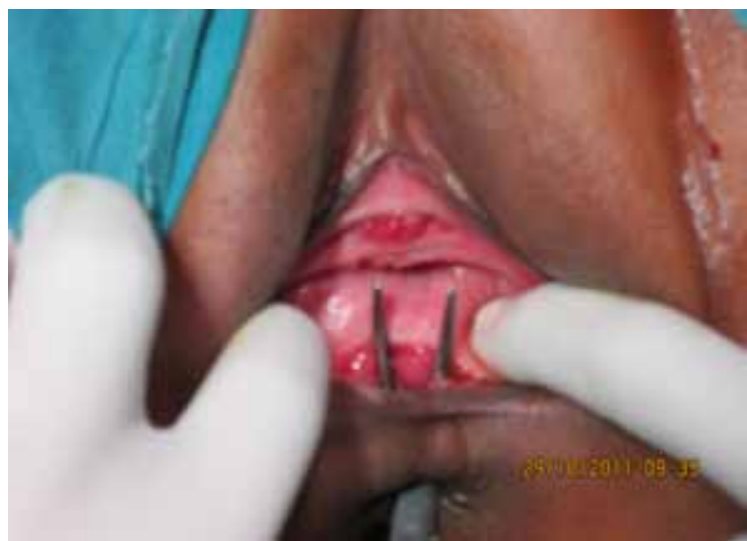

Figure 6. Rent was felt continuous with vagina in the RVF following chronic PID

On examination her vitals and per abdomen examination were normal. On per speculum examination cervix looked congested and with profuse discharge but no bleeding. On per vagina examination there was a small rent about $2 \times 2 \mathrm{~cm}, 1.5 \mathrm{~cm}$ above the introitus (Fig 5) through which stool was coming out. Her uterus and adnexa were normal. On per rectal examination the rent was felt continuous with vagina (Fig 6), without any other rectal anomalies.
Her hematological investigations were normal, last CD4 count was 509. The fistula was repaired transvaginally in three layers with 3-0 vicryl, namely rectal mucosa and submucosa in first layer, muscular wall and serosa in the intervening layer and vaginal wall in the third layer. Though her first post operative period was uneventful, she complained of passage of flatus and faeces through vagina from the second post operative day. She was treated with intravenous antibiotics. As the repairing was failed, an anoscope sigmoidoscopy, fistula biopsy and endoanal ultrasound have been planned to evaluate internal and external sphincters.

\section{COMMENT}

Although there are many different causes of RVF, obstetric trauma is the most common (88\%), usually arise as a complication of a repaired fourth-degree perineal tear $(5 \%$ of all vaginal deliveries result in 3rd or 4th degree tear, 1-2\% of those develop RVF). The fistula presents immediately post-partum from failed recognition of 4 th degree tear or in 7-10 days after apparently normal repair. Risk factors for development of a RVF after vaginal deliveries include prolonged labor, difficult forceps delivery, shoulder dystocia, and a midline episiotomy. ${ }^{1}$

RVF may develop as a result of direct surgical injury to the rectum or vagina, ischemia, or postoperative infection $^{1}$ as in abdominal or vaginal hysterectomy, LAR, hemorrhoidectomy, rectocele repair, local excision of rectal tumors, restorative proctocolectomy with ileal J-pouch etc. RVF may also develop from penetrating or blunt trauma (including coital injury). A RVF developed following gynecological or surgical trauma can be aggravated by the presence of extensive adhesions in the rectovaginal septum due to endometriosis, pelvic inflammatory disease, or pelvic malignancy. Vaginal injuries (mostly in the vaginal vault) particularly during sexual intercourse occur in the posterior fornix followed by those in right and left fornices, lower vagina and occasionally the posterior wall, were usually mild and self-limiting that do not require medical attention. ${ }^{2}$

Inflammatory bowel disease and Crohn's disease (second most common cause of RVF), infections (Cystoglandular abscess, Diverticulitis, TB, amoebiasis, schistosomiasis, LGV), pelvic Radiation (in $2 \%$ patients within 2 years of completion of radiation), malignancies (cervix, rectum, anus, vagina, perineum, leukemia, metastatic disease) and neglected pessary in situ are other important causes of RVF. $^{3}$

Venkatesh and colleagues 4 report that approximately $50 \%$ of small fistulae following child birth may heal without operative intervention during the first 6 to 8 weeks postpartum. Hence spontaneous healing may occur with adequate medical treatment such as total parenteral 
nutrition, antibiotics, and long-time fasting. However, surgical repair remains the mainstay for managing complex fistula. Operative procedures include transvaginal, transanal, abdominal and tissue transposition procedures. The main aim of surgical treatment is to preserve sphincter function. And success of the surgical correction depends on correct cause, classification, location $\&$ accessibility of the RVF, condition of surrounding tissues, status of the sphincter and the number of previous repairs. Surgery should be delayed till the tissue is inflamed and infected. In a case of stage IVa cervical cancer, presenting with rectovaginal fistula, rectal diversion may be performed which is followed by chemoradiation.

\section{ACKNOWLEDGEMENT}

We are thankful to all our teachers, faculty members, colleagues, seniors and juniors of Medical College and Hospital, Kolkata for their cooperation, valuable opinion and kind help.

\section{REFERENCES}

1. Causes of Rectovaginal fistula. In: Rock JA, Jones HW, editor TeLinde's Operative Gynecology, Philadelphia, Lippincott, 10th ed; 2008;40:1015-16

2. Omu AE. Vaginal injuries during coitus. Trop J Obstet Gynecol.1991;2:115-8.

3. Powers K, Grigorescu B, Lazarou G, Greston WM, Weber T. Neglected pessary causing a rectovaginal fistula: a case report. J Reprod Med. 2008;53(3):235-7.

4. Venkatesh KS, Ramanujam PS, Larson DM, Haywood MA Anorectal complications of vaginal delivery. Dis Colon Rectum.1989;32(12):1039-41. 